\title{
Dimensões da percepção de qualidade em serviços em uma instituição de ensino superior
}

\author{
Size of perception about quality in services \\ in high level teaching institutes \\ Dimensiones de la percepcion de la calidad de servicios \\ en una institucion de educacion superior
}

O presente estudo teve como objetivo identificar, a partir de dois modelos teóricos sobre qualidade em serviço, aquele que melhor representasse a realidade dos serviços prestados por uma Instituição de Ensino Superior. Para tanto foi realizado uma pesquisa de campo com uma amostra de 100 estudantes desta instituição, a partir da identificação das possíveis variáveis envolvidas no construto da percepção de qualidade, resultante da análise de referencial teórico. Foi realizada Análise Fatorial para se determinar as dimensões e os possíveis variáveis presentes na composição deste construto. A partir dos resultados obtidos pela análise foi possível concluir que o modelo de Gronroos (1995), com duas dimensões apresenta uma condição mais adequada à realidade desta Instituição de Ensino Superior.
This study aimed to identify, from two theoretical models on quality service which best represent the reality of the services provided by an institution of higher education. A research was conducted with a sample of 100 students of this institution, to identify possible variables involved in the perception of quality construction, resulting from the analysis of the theoretical framework. Factor analysis was performed to determine the dimensions and possible variables present in the composition of this construct. From the results obtained by the analysis it was concluded that the model Gronroos (1995), with two dimensions presents a better condition to the reality of this institution of higher education.
Este estudio tuvo como objetivo identificar, a partir de dos modelos teóricos sobre calidad en servicio, al que mejor representa la realidad de los servicios prestados por una institución de educación superior. Se realizó un estudio de campo con una muestra de 100 estudiantes de esta institución, a partir de la identificación de las posibles variables involucradas en la percepción de calidad de construcción, resultantes del análisis del marco teórico. Se realizó un análisis Factor para determinar las dimensiones y las posibles variables en la composición de esta construcción. A partir de los resultados obtenidos por el análisis se concluyó que el modelo de Grönroos (1995), con dos dimensiones tiene una condición más adecuada para la realidad de esta institución de educación superior.
Palavras-chave: Qualidade em Serviços, Modelos de qualidade, Dimensões de Qualidade.

\section{Autor:}

\section{Dimas Ferreira Vidal}

Mestrado em Administração e Desenvolvimento Empresarial pela UNESA, Especialista em Administração de Marketing pela UCAM, Economista pela UFRRJ e Administrador pela UNESA. Professor e Pesquisador da Universidade Estácio de Sá-UNESA. Brasil.

E-mail:dimasvidal@yahoo.com.br.

\section{introdução}

A competição econômica mundial tem se acirrado, particularmente na última década, fenômeno este genericamente denominado "Globalização". Dentre os diversos fatores que vem contribuindo para este fenômeno, pode-se destacar a diminuição das barreiras nacionais ao comércio exterior, graças particularmente ao papel da organização mundial de comércio, organismo internacional responsável por regulamentar as relações comerciais entre as nações livres, o fortalecimento, nas últimas décadas, dos quatro grandes países emergentes no panorama econômico internacional (Brasil, Rússia, China e Índia), acentuado por uma estagnação econômica dos países desenvolvidos, tanto na América do Norte, quanto na europa. este acirramento competitivo tem promovido o que em economia é denominado "especialização internacional do trabalho", ou seja, os países envolvidos são levados a se dedicarem aquelas atividades econômicas em que possuem maiores vantagens competitivas. já há algumas décadas o nível das atividades industriais, nos países desenvolvidos, vem apresentando taxas de crescimento negativas, substituídas pela força de trabalho barata dos países asiáticos. Até países como o Brasil tiveram que abrir mão de parte de sua indústria pela falta de condição econômica de competir com chineses, coreanos, etc.

Neste contexto, o setor de serviço tem assumido um papel fundamental na manutenção ou no crescimento da atividade econômica na maior parte destes países. A expansão do setor de serviços na composição da atividade econômica tem promovido um maior interesse por parte dos pesquisadores, nas pesquisas relacionadas às suas particularidades, destacando neste trabalho, pesquisas relacionadas com a "qualidade em Serviços". essa foi a motivação para a definição de um modelo teórico mais adequado que permitisse a identificação de dimensões e atributos de qualidade de serviços aplicados à realidade das Instituições Particulares de Ensino Superior. Para tanto, foi realizado um estudo quantitativo, confrontando os modelos de Gronroos (1995) 
e o modelo de parasuraman,zeithaml, berry (1991) conhecido como Servqual, com este propósito.

\section{Referencial teórico}

\section{Serviços. Conceitos e definições}

Na nova ótica do mercado, na qual a competição deixou de ser local e passou a ser global, a concorrência intensa levou as empresas a conquistarem os clientes (grÖNrooS, 1993), saindo de uma situação de passividade em relação a eles, em um outrora mercado caracterizado pela grande demanda e pouca oferta de produtos e serviços.

para atrair os clientes para si as organizações objetivam diferenciar-se em relação aos concorrentes. No caso de prestação de serviços, a qualidade é um aspecto recorrente na literatura como uma competência essencial no desenvolvimento de vantagem competitiva (grÖNrooS, 1995, forNell, 1992, e

PARASURAMAN; ZEITHMAL; BERRY, 1991,).

zeithaml e bitner (2003) apud rocha e oliveira (2003) definem serviços como atos e processos destinados à produção de algo intangível, necessário ao consumidor, ou seja, uma atividade econômica cujo resultado, em sua maioria, não apresenta um produto físico. Esta mesma definição já havia sido destacada por Kotler e Armstrong (2000) e por Gronroos (1995).

Para este último, diferente de produtos que possuem características e atributos mais tangíveis, os serviços são mais percebidos pelos processos que os produzem. Gronroos (1995) definiu ainda, quatro características para o serviço, características estas que contribuíram para a formulação de seu modelo de qualidade:

- Intangibilidade - O resultado final de um serviço, o qual poderia ser chamado de produto do serviço, não apresentam, em sua maioria, características ou atributos muito tangíveis.

quais seriam os atributos de um atendimento médico a uma consulta, ou de uma sessão de cinema, ou ainda de uma formação em um curso superior em administração, que pudessem servir de parâmetros para uma análise de conformidade.

- Inseparabilidade - Na maioria das vezes o produto de um serviço é consumido durante seu processo de produção, ou seja, a produção e o consumo não estão separados, e em boa parte das vezes com a participação ativa do proprio consumidor, assumindo este, as vezes um papel importante no processo, como é o caso do serviço prestado por uma Instituição de curso Superior.

- Variabilidade - Em função da característica da inseparabilidade, é bem mais difícil padronizar os processos de produção do serviço, visto que podem, em cada execução do processo, estarem presentes variáveis exógenas.

- perecibilidade - o produto de serviço, em sua maioria, não pode ser estocado ou armazenado, visto que seu consumo é inseparável de sua produção.

\section{Qualidade em Serviço}

gronroos (1995) destaca que a qualidade de serviço é o resultado da avaliação entre as expectativas do consumidor frente ao serviço prestado. A mesma visão é compartilhada por parasuraman, zeithaml, e berry (2003). estes autores consideram que a qualidade percebida por um consumidor de um serviço é definida pela comparação com suas expectativas ao serviço esperado. Estas expectativas poderiam ser construídas pelas experiências deste consumidor, em suas relações de consumo, pelas perspectivas construídas pela propaganda e publicidade do prestador de serviço, ou ainda por padrões socialmente cons- truídos em um processo cultural. zethaml e bitner (2000) apud rocha e oliveira (2003) destacam que a qualidade percebida pelo consumidor é resultante de seu julgamento relacionado ao seu conceito de excelência. Diferente da qualidade objetiva, fatores como atitude, bem estar e performance é que determinarão seu julgamento. Grönroos (1990) e Fornell (1992) defendem que o importante é como a qualidade é percebida pelo cliente.

Diante disto, há que se fazer uma distinção entre a qualidade dos serviços e a satisfação dos clientes. É o que afirmam Lovelock e Wirtz(2006) e Bateson (2003). Segundo esses autores a qualidade do serviço deriva de avaliações cognitivas de longo prazo por parte dos clientes, acerca da entrega do serviço.o conceito de satisfação está relacionado com o resultado obtido naquele serviço específico. A satisfação do consumidor é uma reação de curta duração ao desempenho específico de um serviço (LOVELOCK E WRIGHT, 2003).

\section{O Modelo de Qualidade de Gronroos}

Gronroos (1995) fornece um modelo genérico de qualidade em serviço a partir de duas dimensões: a qualidade técnica e a qualidade funcional. A qualidade técnica está ligada ao produto do serviço e está relacionada á capacidade do consumidor perceber os atributos a partir da entrega do resultado do serviço. Segundo este autor, esta dimensão pode ser avaliada de forma mais objetiva pelo consumidor a partir de uma série de características esperadas pelo serviço ofertado. Ainda segundo Gronroos (1995), a qualidade funcional está ligada ao processo de produção do serviço, ao ambiente, ao atendimento e ao conforto ou desconforto que o consumidor experimenta. Ainda segundo este autor, dado suas características subjetivas, esta dimensão é geralmente avaliada a partir de suas sensações e expectativas ao longo do processo.

Nafigura 1 é apresentado o processo da qualidade per cebida definidas por Verruck et al.(2008).

Figura 1

Processo da construção da qualidade percebida

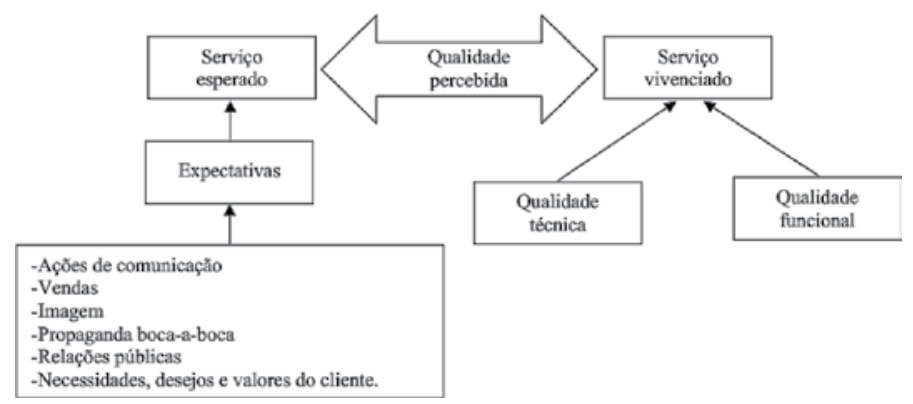

Fonte: Adaptado por Verrucket al. (2008) a partir de Gronross (1995)

As duas dimensões do modelo, qualidade funcional e a qualidade técnica serão resultantes do processo de comparação entre a qualidade esperada gerada pelas expectativas construídas através da propaganda e publicidade, da promoção de vendas, da propaganda boca a boca, pelos mecanismos de comparação com as experiências do consumidor do serviço, e o serviço vivenciado, tanto sob o aspecto do processo quanto do resultado do serviço.

\section{Modelo de qualidade SERVCAL}

parasuraman, zeithaml, e berry (1991) desenvolveram um modelo genérico de qualidade de serviço denominado ServcAl. Este modelo, constituído de cinco dimensões, buscava de forma mais clara definir um conjunto de atributos ou características, 
ligadas a estas dimensões, que pudessem servir de parâmetro na avaliação da qualidade percebida a partir das expectativas do consumidor de serviço. As cinco dimensões são:

- Confiabilidade - Toda e qualquer relação de consumo envolve algum nível de risco de se não obter aquilo que se espera sobre o produto ou serviço. o consumidor, ao optar por uma marca ou empresa nesta relação de consumo, está em boa parte das vezes, buscando uma relação de confiabilidade. Esta dimensão representa a busca da garantia de que se seja entregue aquilo que efetivamente ele buscava.

- Tangibilidade - Os autores destacam a importância das questões tangíveis, tais como aspecto físico das instalações, condições climáticas dos ambientes, equipamentos e infra-estrutura operacional, pessoal qualificados, visto que sobre este aspecto, fica mais fácil, para o consumidor identificar atributos da qualidade esperada no processo da prestação de serviço.

- Responsabilidade e presteza - Está relacionado à visão que o consumidor tem do papel dos funcionários da empresa prestadora de serviço, ou seja, o quanto estes funcionários se mostram responsáveis pela qualidade, presteza e conforto do cliente no processo.

- Segurança - Similar à dimensão de confiabilidade, no entanto, difere deste por estar relacionado com os riscos envolvidos em sua participação ao longo do processo. Na busca por segurança, aspectos ligados às questões financeiras, ao ambiente, às pessoas e as questões legais e morais são considerados importantes, pelo consumidor, em sua relação de consumo de serviço.

- Empatia - Está relacionada ao atendimento dos funcionários da prestadora de serviço ao cliente, à atenção e à boa vontade destes funcionários no atendimento às necessidades deste cliente.

fitzsimmons e fitzsimmons (2005), a partir do modelo de parasuraman, zeithaml, e berry (1991) desenvolveram um quadro representando o processo da construção da qualidade percebida (figura 2). A partir das cinco dimensões de qualidade, e em função experiências, necessidades e informação, o consumidor faz uma comparação entre o serviço esperado e o serviço percebido.

\section{Figura 2 \\ Processo de construção da qualidade percebida pleo modelo SERVCAL}

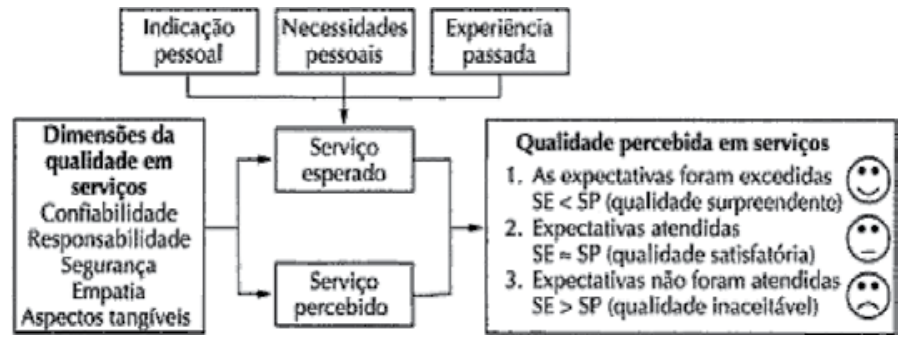

Fonte: Fitzsimmons e Fitzsimmons (2005, p. 147)

Se o cliente tiver suas expectativas excedidas, ou seja, a qualidade percebida for maior que suas expectativas, ele classificará o serviço com muita qualidade. Se suas expectativas forem atendidas, ou seja, a qualidade percebida for exatamente igual as suas expectativas, ele classificará o serviço como de qualidade. E por último, se suas expectativas não forem atingidas, ou seja, se a qualidade percebida for inferior à suas expectativas, ele irá considerar a qualidade inaceitável.
Aparentemente, os dois modelos, de gronroos (1995) e de parasuraman, zeithaml, e berry (1991), seguem os mesmos princípios, sendo que o modelo ServcAl busca ser mais específicos na definição das características e atributos que servem de base para medir a qualidade percebida de serviços.

Nesta pesquisa, estes dois modelos serão testados para determinar aquele que se mostrar mais adequados, como um modelo de qualidade percebida dos serviços de uma Instituição de ensino Superior.

\section{Metodologia}

O objetivo principal da presente pesquisa foi encontrar um modelo teórico que permitisse a identificação das dimensões e atributos de qualidade de serviços aplicados à realidade da Instituição de Ensino Superior em estudo. Para tanto, foi realizado um pesquisa confrontando o modelo de Gronroos (1995) composto por duas dimensões e o modelo ServcAl de parasuraman, zeithaml, berry (1991) composto de cinco dimensões.

de caráter descritivo, a presente pesquisa,tem abordagem quantitativa. teve como meios de investigação uma pesquisa bibliográfica para a formulação dos fundamentos teóricos que deram a base para a identificação das variáveis que fizeram parte de uma pesquisa de campo. Para tanto, foi utilizado um questionário estruturado, fazendo uso de uma escala Likert, composto por vinte sete questões, cada uma delas relacionada a uma das variáveis definidas, resultante da combinação dos dois modelos do estudo (GIL, 1999, COOPER; SCHINDLER, 2001).

portanto, a estratégia metodológica consistiu em identificar variáveis que pudessem estar relacionadas às dimensões dos dois modelos e fazer uso de Análise Fatorial para a identificação e possível confirmação, das dimensões de variabilidade presentes. Ao realizar o agrupamento em fatores ou dimensões, a Análise Fatorial faz o agrupando de variáveis que possuem correlação entre si, estabelecendo algum nível de dependência entre elas, e separando em grupos aquelas que não a possuem. Neste processo, a Análise fatorial também busca destacar aquelas variáveis que tem um poder muito baixo de explicação pela dimensão relacionada, através de uma correlação muito baixa. esta técnica estatística permite, não só determinar as dimensões envolvidas (variáveis latentes) no estudo, mas também é capaz de estabelecer o nível de importância que cada variável possui na composição desta dimensão, bem como afastar aquelas com baixo poder de influência da mesma (CORRAR et al., 2011).

Coleta, procedimentos da pesquisa e tratamento dos dados

Os questionários foram entregues diretamente a 100 (cem) alunos dos cursos de Administração, Recursos Humanos e Sistema da Informação de uma Instituição de Ensino Superior localizada no estado do rio de janeiro. foi realizada, para o grupo pesquisado, uma apresentação dos objetivos da pesquisa e respondidas algumas dúvidas que se apresentaram. do total de respondentes, foi descartado somente um questionário que apresentava questões não respondidas.

A partir da revisão da literatura e dos objetivos da presente pesquisa, foram identificadas 27 (vinte sete) variáveis relacionadas às dimensões dos dois modelos envolvidos (groNrooS 1995,

PARASURAMAN; ZEITHAML; BERRY, 1991).

Atr01 - programa do curso mantido pela universidade;

ATR02 - Domínio do conteúdo pelos professores;

ATR03 - Aulas interessantes ministradas pelos professores; Atr04 - domínios adquiridos pelo aluno;

ATR05- Formação acadêmica do professor; 
ATR06 - Recursos pedagógicos disponibilizados pelo professor; Atr07 - processo de avaliação;

Atr08 - tecnologia de disciplinas à distância;

ATR09 - Cumprimento de programa pelo professor;

Atr10 - consideração dos interesses do aluno por parte da instituição;

Atr11 - disponibilidade do coordenador;

Atr1 2- reputação da instituição vista pelo aluno;

Atr1 3-As instalações do campus;

Atr14 - A imagem da universidade;

ATR15 - Compreensão do professor pelas questões do aluno;

Atr1 6- disposição dos gestores em resolver as questões dos

alunos;

ATR17 - Habilidade do coordenador em resolver as questões dos alunos;

Atr18 - preocupação da gestão com a qualidade;

Atr1 9- calendário acadêmico;

Atr20 - compreensão do coordenador às questões do aluno;

ATR21 - Atendimento do funcionário;

Atr22 - Atendimento da secretaria;

ATR23 - Horário de aula;

ATR24- Conforto em sala de aula;

ATR25 - Sistema de informação acadêmica;

Atr26 - equipamentos de apoio acadêmico;

Atr27 - critérios adotados para estabelecer preços.

para a realização da análise fatorial foram selecionados os testes e recursos abaixo relacionados, fazendo uso do software SPSS 17 (corrAr et al., 2011).

a) Determinante

o determinante necessita ser superior a 0,00001.

Se o determinante for zero, não há solução analítica, pois ele define se determinada matriz quadrada terá inversa.

\section{-Meier-Olkin}

b) Testes KMO - Kaiser-

$O$ teste KMO mede a adequação da amostra à aplicação da Análise fatorial. o resultado obtido deve ser superior a 0,70 (70\%), sendo inadequada quando inferior a $0,50(50 \%)$. O KMO testa se há variáveis suficientes em cada fator.

c) Teste de esfericidade de Bartlett (Bartlett's test of sphericity)

o teste de barttlett's ve-

rifica se a matriz de correlação é uma matriz identidade. o objetivo deste teste é também verificar a adequação da amostra à aplicação da Análise fatorial. para que seja possível a aplicação desta técnica, é recomendável que o valor de significância obtido não ultrapasse a $0,05(0,5 \%)$.

d) Comunalidade

Fonte: Dados obtidos do SPSS 17
A comunalidade é a proporção da variância de uma variável observada que é explicada pelos fatores extraídos. As variáveis com comunalidades menores do que 0,50 (50\%) não exibem explicação suficiente.

e) Extração de Fatores com autovalor (Eigen values) maior que 1 (um)

Como requisito para a extração de fatores, será utilizado como critério, os fatores que tiverem autovalor maior que 1.

f) Método Principal Axis Factoring

por se tratar de Análise fatorial, será utilizado o método de extração denominado Principal Axis Factoring.

\section{g) Método de rotação Varimax}

O método de rotação da matriz será o Varimax.

h) Ordenado pelo maior valor e supressão de valores absolutos menores que $30 \%$.

\section{Apresentação dos resultados da pesquisa}

A partir da coleta dos dados, os mesmos foram inseridos no SpSS e processados com o recurso de "fator Analysis" desta ferramenta. Os resultados e procedimentos são apresentados abaixo.

$\mathrm{Na}$ figura 1 são apresentados os testes KMO - KaiserMeier-Olkin, e de esfericidade de Bartlett's, cuja finalidade, para os dois testes, é determinar a adequação da amostra à técnica de Análise fatorial. São apresentados, também, os valores de comunalidade, cujo objetivo é apresentar a proporção de cada variável observada que é explicada pelos fatores extraídos.

Figura 3

Testes de KMO e Bartlett's

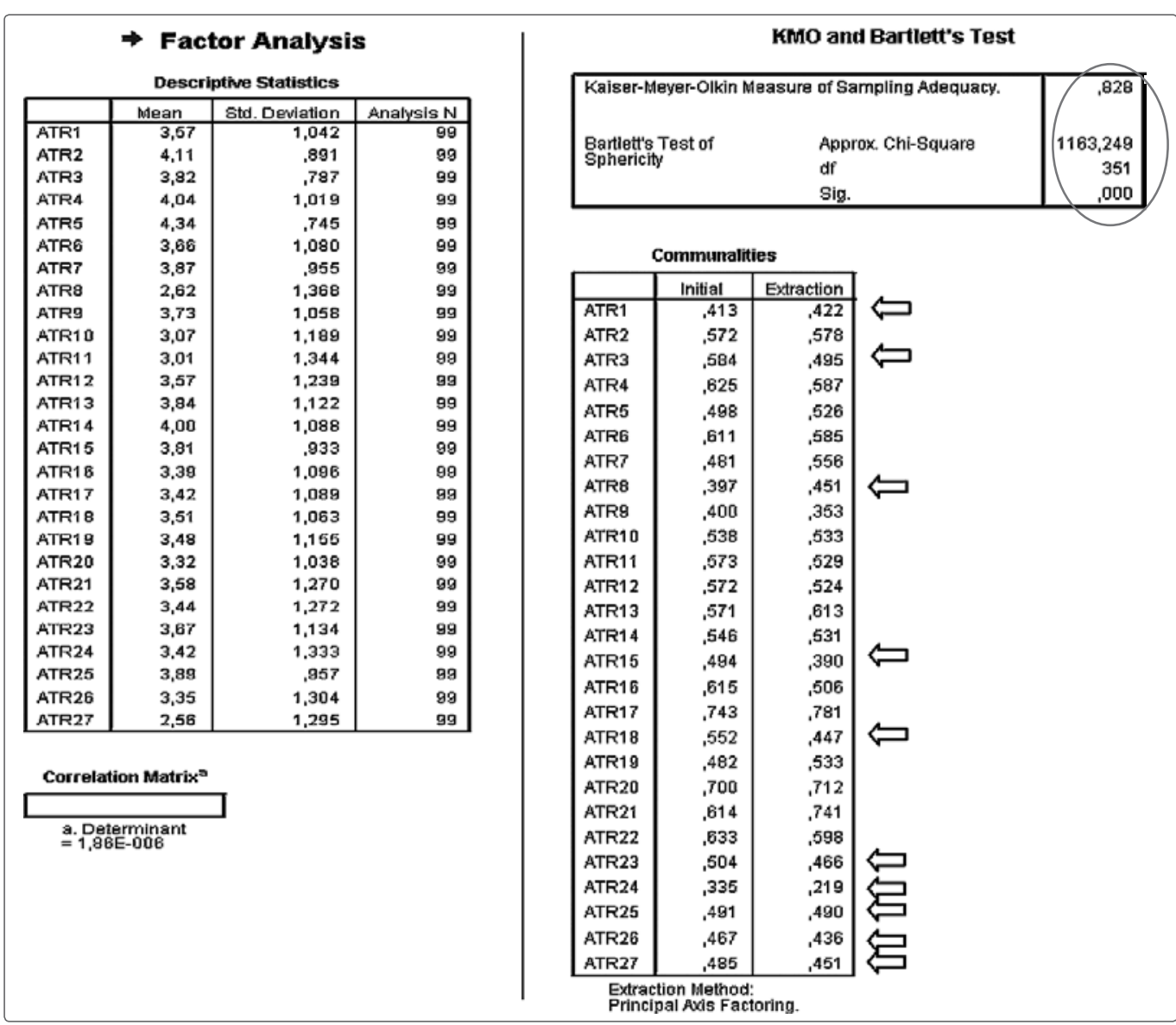


Os dados passaram pelo teste de $\mathrm{KMO}$, pois foi obtido um valor 0,828 de KMO, acima, portanto dos 0,70 necessários para este teste. Os dados também passaram pelo teste de esfericidade de Bartlett's, pois o teste não ultrapassou os 0,05 de significância, mostrando que, em ambos os casos, existe uma condição adequada para a aplicação da Análise fatorial.

Com relação a Cumunalidade, verificou-se dez variáveis com valores abaixo de 50\% (cinqüenta) de comunalidade. Isto nos sugere que algumas das variáveis observadas não apresentam uma representatividade adequada (corrAr et al., 2011).

\begin{tabular}{|c|c|c|c|c|c|c|}
\hline \multicolumn{7}{|c|}{ Total Variance Explained } \\
\hline \multirow[b]{2}{*}{ Factor } & \multicolumn{3}{|c|}{ Initial Eigenvalues } & \multicolumn{3}{|c|}{ Extraction Sums of Squared Loadings } \\
\hline & Total & $\%$ of Variance & Cumulative \% & Total & $\%$ of Variance & Cumulative $\%$ \\
\hline 1 & 8,634 & 31,977 & 31,977 & 8,178 & 30,288 & 30,288 \\
\hline 2 & 1,884 & 6,976 & 38,954 & 1,424 & 5,273 & 35,562 \\
\hline 3 & 1,636 & 6,058 & 45,011 & 1,183 & 4,381 & 39,943 \\
\hline 4 & 1,518 & 5,622 & 50,633 & 1,048 & 3,880 & 43,823 \\
\hline 5 & 1,387 & 5,136 & 55,769 &, 921 & 3,411 & 47,234 \\
\hline 6 & 1,232 & 4,564 & 60,333 & .759 & 2,812 & 50,046 \\
\hline 7 & 1,022 & 3,786 & 64,119 & .539 & 1,997 & 52,042 \\
\hline 8 & ,968 & 3,585 & 67,704 & & & \\
\hline 9 & ,875 & 3,242 & 70,946 & & & \\
\hline 10 & .846 & 3,135 & 74,081 & & & \\
\hline 11 & .766 & 2,836 & 76,918 & & & \\
\hline 12 & .681 & 2,521 & 79,438 & & & \\
\hline 13 & 638 & 2,364 & 81,802 & & & \\
\hline 14 & .611 & 2,262 & 84,064 & & & \\
\hline 15 & .586 & 2,170 & 86,234 & & & \\
\hline 16 & .551 & 2,039 & 88,273 & & & \\
\hline 17 & .454 & 1,683 & 89,957 & & & \\
\hline 18 & .411 & 1,522 & 91,479 & & & \\
\hline 19 & .373 & 1,382 & 92,861 & & & \\
\hline 20 & .350 & 1,296 & 94,157 & & & \\
\hline 21 & .314 & 1,163 & 95,320 & & & \\
\hline 22 & .271 & 1,002 & 96,322 & & & \\
\hline 23 & .263 &, 973 & 97,295 & & & \\
\hline 24 & .235 & .869 & 98,163 & & & \\
\hline 25 & ,193 & .713 & 98,877 & & & \\
\hline 26 & 164 & .607 & 99,483 & & & \\
\hline 27 & .140 & .517 & 100,000 & & & \\
\hline
\end{tabular}

Extraction Method: Principal Axis Factoring.

Figura 5

Matriz de Fatores Rotacionada

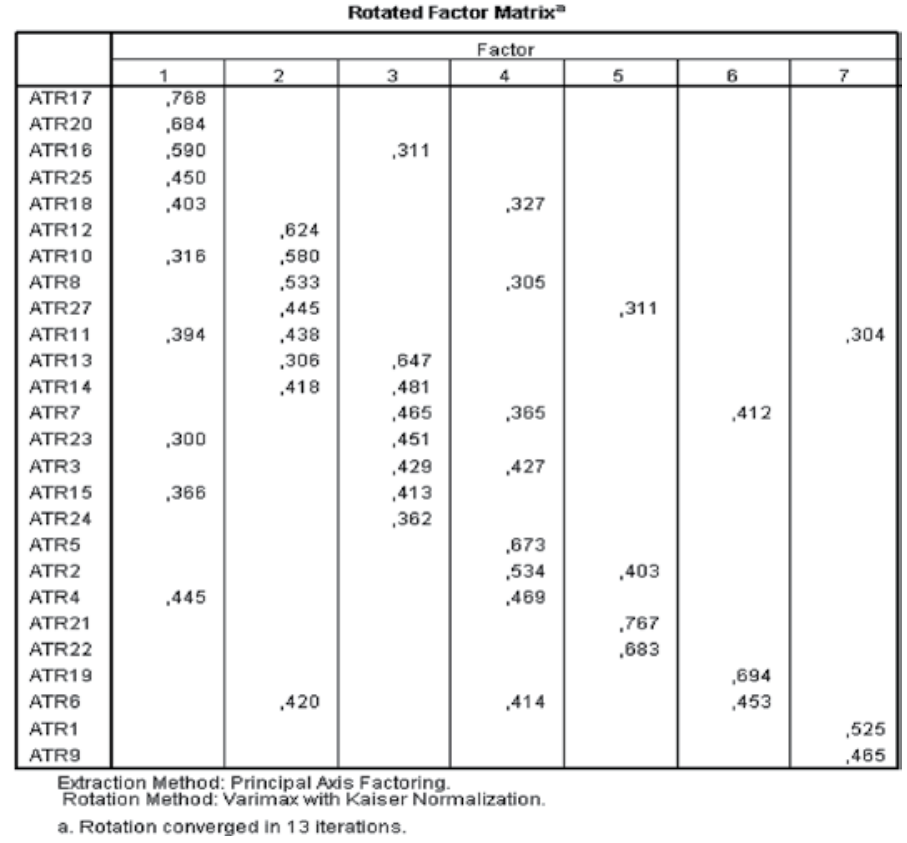

Fonte: Dados obtidos do SPSS 17
O passo seguinte foi realizar a extração dos fatores através do método de Análise Fatorial e determinar a matriz de fatores rotacionada pelo método Varimax. Na figura 4 e 5 são apresentados os dados relacionados a essas suas ações.

O resultado da extração apresenta 7 (sete) fatores com autovalor maior que 1 (um). É importante verificar que os sete fatores apresentam aproximadamente $64 \%$ (sessenta e quatro por cento) da variância explicada, um valor consideravelmente alto.

O resultado da matriz de fatores rotacionada pelo método Varimax apresentou diversas variáveis que possuem autovalores maiores que $30 \%$ (trinta por cento) em mais de um fator. Este fato demonstra que estas variáveis possuem uma correlação significativa com mais de uma dimensão. Neste caso, o procedimento metodológico consiste em ir retirando, um a um, os fatores da nossa análise, até que se obtenha variáveis que efetivamente tenham correlação alta somente com um fator (CORRAR et al., 2011). Neste processo chegamos aos resultados apresentados nas figuras 5 e 6 com somente dois fatores (dimensões).

Figura 5

Dados de extração com dois fatores

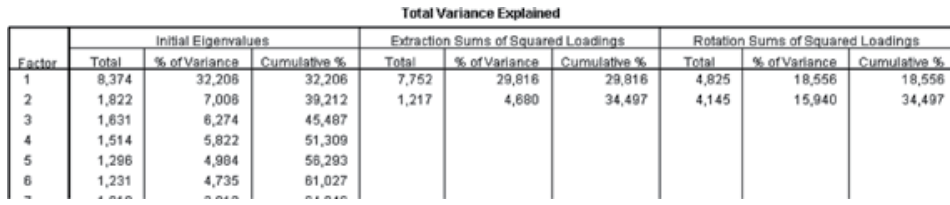

Figura 6

Matriz rotacionada com autovalores de cada variável

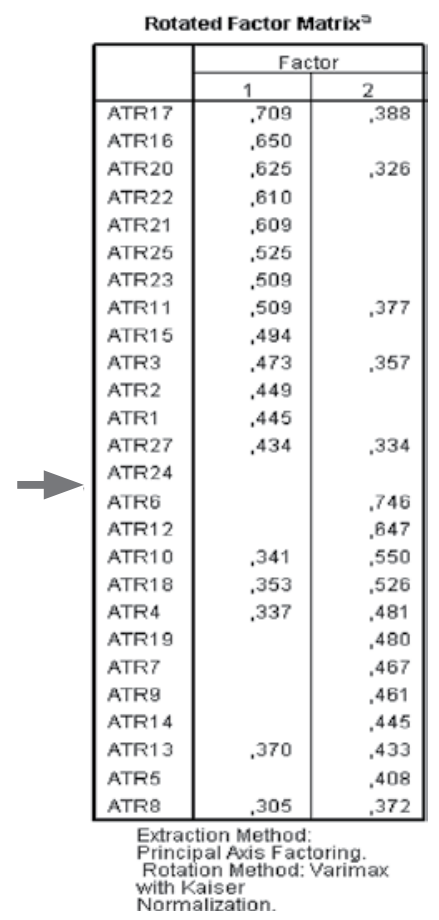

Fonte: Dados obtidos do SPSS 17

A partir destes resultados, pode-se verificar que ainda há 10 (dez) variáveis que possuem autovalor maior que 30\% (trinta por cento) nos dois fatores obtidos. Neste caso, o proce- 
dimento consiste em retirá-los do estudo e realizar uma nova análise (corrAr et al., 2011). foram retiradas do estudo as seguintes variáveis: ATR17 - Habilidade do coordenador em resolver as questões dos alunos; Art20 - compreensão do coordenador às questões do aluno; Atr11 - disponibilidade do coordenador; Atr3 - aulas interessantes ministradas pelo professor; ATR27 - Critérios adotados para estabelecer preços; Atr10 - consideração dos interesses do aluno por parte da instituição; Atr18 - preocupação da gestão com a qualidade; Atr4 - domínio adquirido pelo aluno; Atr13 - Instalações do campus; Atr8 - tecnologia de disciplina a distância.

Ao realizar novamente da análise, a variável Atr14 - A imagem da universidade perante o aluno, e Atr 2 - domínio do conteúdo pelo professor, também apresentaram autovalor maior que $30 \%$ (trinta por cento) nos dois fatores. Portanto também foram retirados.

Figura 7

Matriz rotacionada com a retirada de variáveis

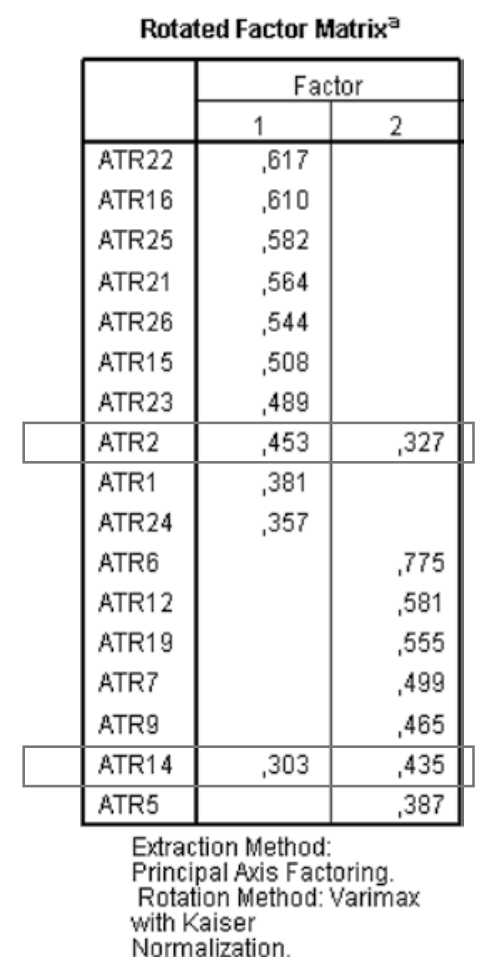

Fonte: Dados obtidos do SPSS 17

\section{Resultados finais}

Nas figuras 8, 9 e 10 são apresentados os resultados da análise final pelo SPSS. O total de variância explicada pelos fatores obtidos foi de $33,78 \%$, valor este que pode ser considerado baixo, levantando a hipótese da existência de outras variáveis importantes que não fizeram parte do estudo.

Verifica-se, também, que nesta última etapa da análise, algumas das variáveis observadas possuem comunalidades abaixo de $0,50(50 \%)$. Neste caso, os dados obtidos sugerem que estas variáveis não possuem um nível de explicação suficiente, indicando a possível necessidade de trocá-las por outras variáveis com maior pode de explicação (CORRAR et al., 2011) .

A tabela da matriz rotacionada, apresentada na figura 10 , indicam as variáveis que possuem os maiores autovalores em cada uma das dimensões encontradas.

Figura 8

Total de variância explicada pelos fatores

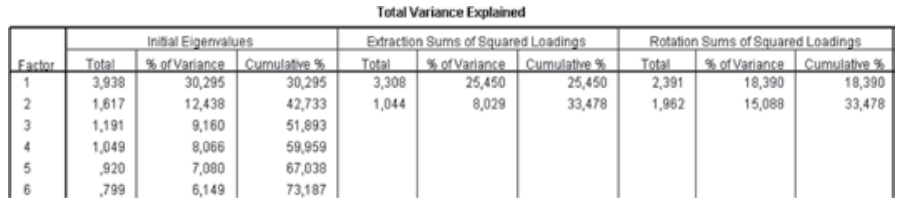

Fonte: Dados obtidos do SPSS 17

Figura 9

Resultados da análise final

Factor Analysis
\begin{tabular}{|l|r|r|r|}
\hline \multicolumn{4}{c|}{ Descriptive Statistics } \\
\hline ATR1 & Mean & Std. Deviation & Analysis N \\
ATR5 & 4,57 & 1,042 & 99 \\
ATR6 & 4,34 &, 745 & 99 \\
ATR7 & 3,66 & 1,080 & 99 \\
ATR12 & 3,87 &, 955 & 99 \\
ATR15 & 3,57 & 1,239 & 99 \\
ATR16 & 3,81 &, 933 & 99 \\
ATR19 & 3,39 & 1,096 & 99 \\
ATR21 & 3,48 & 1,155 & 99 \\
ATR22 & 3,58 & 1,270 & 99 \\
ATR23 & 3,44 & 1,272 & 99 \\
ATR24 & 3,67 & 1,134 & 99 \\
ATR25 & 3,42 & 1,333 & 99 \\
ATR2 & 3,89 &, 957 & 99 \\
\hline
\end{tabular}

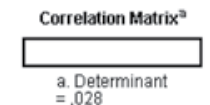

KMO and Bartlett's Test
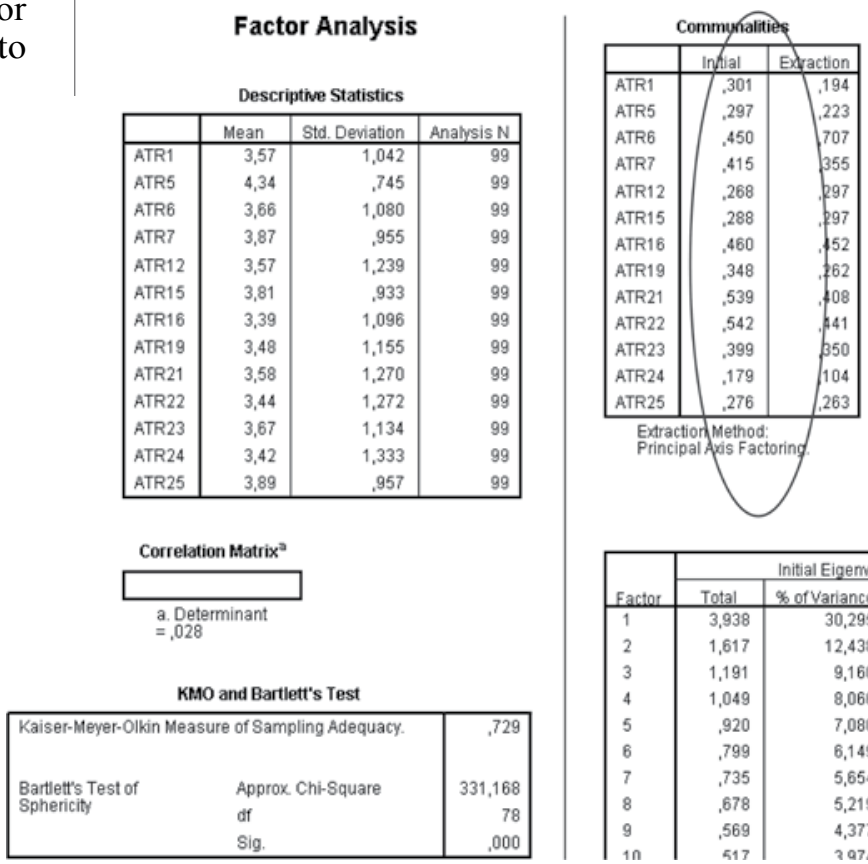

\begin{tabular}{|l|r|r|r}
\hline \multirow{2}{*}{ Factor } & \multicolumn{3}{|c}{ Initial Eigenvalues } \\
\cline { 2 - 4 } & \multicolumn{1}{|c|}{ Tota| } & \%orvariance & Cumulative \% \\
\hline 1 & 3,938 & 30,295 & 30,295 \\
2 & 1,617 & 12,438 & 42,733 \\
3 & 1,191 & 9,160 & 51,893 \\
4 & 1,049 & 8,066 & 59,959 \\
5 &, 920 & 7,080 & 67,038 \\
6 &, 799 & 6,149 & 73,187 \\
7 &, 735 & 5,654 & 78,841 \\
8 &, 678 & 5,219 & 84,060 \\
9 &, 569 & 4,377 & 88,436 \\
10 & 517 & 3974 & a) $\Delta 10$
\end{tabular}

Figura 10

Resultado da matriz rotacionada

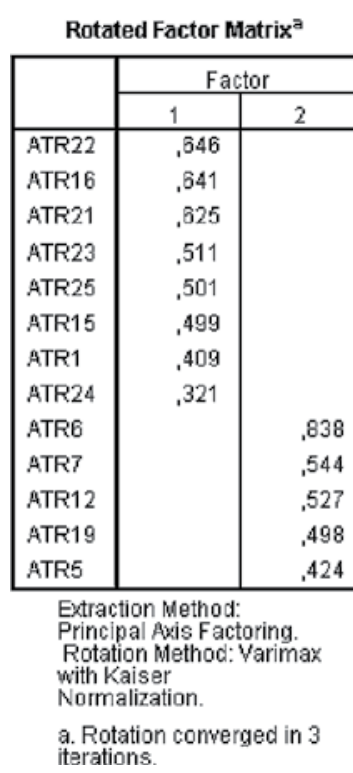

Fonte: Dados obtidos do SPSS 17 
As seguintes variáveis estão presente no fator 1 (um): Atr22 - Atendimento da secretaria - Autovalor: 0,646; Atr16 - disponibilidade dos gestores em resolver as questões dos alunos - Autovalor: 0,641;

ATR21 - Atendimento do funcionário - Autovalor: 0,625; ATR23 - Horários das aulas - Autovalor: 0,511; ATR25 - Sistema de Informação Acadêmica - Autovalor: 0,501 ;

ATR15 - Compreensão do professor pelas questões do aluno - Autovalor: 0,499;

Atr1 - programa do curso mantido pela universidade

- Autovalor: 0,409;

ATR24 - Conforto em sala de aula - Autovalor: 0,321.

Para o fator 2 (dois), foram observadas as seguintes variáveis:

Atr6 - recursos pedagógicos disponibilizados pelo professor - Autovalor: 0,838;

ATR7 - Processo de avaliação utilizado pelo professor - Autovalor: 0,544;

Atr12 - reputação da instituição vista pelo aluno -

Autovalor: 0,527;

Atr19 - calendário acadêmico - Autovalor: 0,498;

ATR5 - Formação acadêmica do professor - Autovalor: 0,424 .

\section{Considerações finais}

com relação aos resultados obtidos pela Análise fatorial, pode-se concluir que, pelo menos em parte, o objetivo principal foi atendido, pois foi possível identificar duas dimensões e treze variáveis presentes na composição da percepção de qualidade de serviço de Instituições de ensino Superior em estudo. pode-se concluir também que o modelo de gronroos (1995) é, aparentemente, é o modelo mais próximo da realidade da Instituição de ensino Superior pesquisada. Ao analisar os agrupamentos formados, o conjunto de variáveis agrupadas no fator 1, estas representariam a dimensão funcional de seu modelo, e as variáveis agrupadas no fator 2 representariam a Dimensão Técnica.

Ainda segundo os resultados, a Dimensão funcional, dado o número maior de variáveis presentes, indica um grau maior de importância desta dimensão na percepção de qualidade por parte de seu cliente, enquanto a variável que representa o papel do professor e de seu conjunto de recursos pedagógicos (dimensão técnica) como a variável isolada mais importante na sua percepção de qualidade de Instituições de ensino Superior. Neste contexto, pode-se afirmar que os processos envolvidos na produção do serviço prestado pela Instituição de ensino Superior, representam, para os alunos desta instituição, os aspectos mais importantes na definição dos atributos responsáveis pela construção de sua percepção de qualidade. esta constatação já era esperada, visto que, ao contrário da maioria dos serviços, os clientes de uma Instituição de ensino Superior mantém uma relação de consumo do mesmo serviço ao longo de vários anos, evidenciando a importância de atributos de qualidade relacionados à sua participação no processo.

os resultados obtidos devem ser vistos com alguma ressalva, visto que várias variáveis obtiveram uma cumunalidade inferior a 50\%, a pesar de o teste de KMO indicar um alto poder de explicação entre os fatores identificados e as variáveis envolvidas.

\section{Referências Bibliográficas}

BATESON, John E. G., HOFFMAN, K. Douglas. Marketing de serviços. Porto Alegre: Bookman, 2003.

COOPER, Donald, SCHINDLER, Pamela. Método de pesquisa em Administração. $7^{\mathrm{a}}$ ed. Porto Alegre: Bookman, 2001.

CORRAR, Luiz J., PAULO, Edilson, DIAS FILHO, José M. Análise Multivariada para os Cursos de Administração, Ciências Contábeis e Economia. São paulo: Atlas, 2011.

fItzSImmoNS, james A., fItzSImmoNS, mona j. Administração de serviços. $4^{\mathrm{a}}$ ed. São Paulo: Bookman, 2005.

forNell, claes. A national customer satisfaction barometer: the Swedish experience. Journal of Marketing, v. 56, n. 1, p. 6-21, 1992.

gIl, Antônio c. Métodos e Técnicas em Pesquisa Social. $5^{\mathrm{a}}$ ed. São paulo: Atlas, 1999.

grÖNrooS, christian. Marketing: gerenciamento e serviços. rio de janeiro: campus, 1995.

KOTLER, Philip; ARMSTRONG, Gary. Introdução ao marketing. $4^{\mathrm{a}}$ ed. rio de janeiro: ltc, 2000.

LOVELOCK, Christopher, WIRTZ, Jochen. Marketing de serviços: pessoas, tecnologia e resultados. $5^{\mathrm{a}}$ ed. São Paulo: Prentice Hall, 2006.

LOVELOCK, Christopher, WRIGHT, Lauren. Serviços: marketing e gestão.São paulo: Saraiva, 2003.

PARASURAMAN, A..; ZEITHAML, V. A.; BERRY, L. L. Refinement and reassessment of the SERVQUAL Scale. Journal of Retailing, $\mathrm{v}$. 67, n. 4, winter, 1991.

ROCHA, Vítor Andrade G. A.; OLIVEIRA, Andrea da Silva. Avaliando a Qualidade de Serviço: Aplicação da Escala SERVQUAL numa Grande Instituição Bancária. enanpad, Anais..., 2003.

VERRUCK, Fábio; LAZZARI, Fernanda; BAMPI, Rodrigo Eduardo, mueller, Antony peter. Atributos e Dimensões da Qualidade em Serviços: Um Estudo Aplicado em uma Empresa de Transporte Urbano. rio de janeiro, enanpad, Anais..., 2008.

ZEITHAML, V. \& BITNER, M.J.Marketing de Serviços, porto Alegre: Bookman, 2003.

Artigo enviado em 27/11/2014, aceito em 12/12/2014 